\title{
EFFECTS OF GLUCOSE AND BILIRUBIN ON THE KINETIC JAFFE'S AND THE ENZYMATIC METHODS FOR SERUM CREATININE ASSAY
}

\author{
Irena I. Gencheva, \\ Adelaida L. Ruseva
}

Clinical Laboratory

Medical University - Pleven

\section{Corresponding Author:}

Irena I. Gencheva

Clinical laboratory

UMBAL "dr. Georgi Stranski”

8A, Georgi Kochev str.

Pleven, 5800

Bulgaria

Received: March 30, 2015

Revision received: April 03, 2015

\begin{abstract}
Summary
The enzymatic method for the determination of serum creatinine is accepted as one of the most accurate methods in a clinical laboratory. This method was used on a biochemistry auto analyzer (Cobas Integra 400) to determine serum creatinine at the laboratory of University Hospital - Pleven. The characteristics and reliability of this enzymatic method for creatinine were compared with the Jaffe kinetic method. Effects of some interfering substances like bilirubin and glucose on the Jaffe kinetic method and the enzymatic method were compared. Glucose and bilirubin inhibit the reaction between creatinine and alkaline picrate. Glucose slowly reduces picric acid to picrate, while the bilirubin present in a sample is oxidized to biliverdin under alkaline conditions. This leads to a decrease in absorbance at $520 \mathrm{~nm}$. We measured creatinine in serum samples with the enzymatic method and the Jaffe kinetic method in samples divided into four groups: group I -samples without bilirubin and glucose ; group II -samples with high level of glucose; group III - samples with high level of bilirubin, group IVall samples. For Group I, the correlation coefficient obtained by comparing the enzymatic creatinine method and Jaffe's kinetic method was $\mathrm{R}=0.983$. There was a very good agreement between the two methods in terms of correlation coefficient even in the samples with high levels of glucose or bilirubin.
\end{abstract}

Key words: creatinine, enzymatic assay, Jaffe kinetic method, glucose, bilirubin

\section{Introduction}

Several methods for estimation of serum and urinary concentrations of creatinine, most of which are based on the Jaffe's reactions are routinely used in clinical biochemistry laboratories. Using such methods is associated with problems concerning sample analysis, and this could be attributed to the Jaffe's reaction. Numerous chromogenic interfering substances have been documented. Usually, glucose and bilirubin are cited as interfering substances of the Jaffe's based methods [1-3]. Both substances inhibit the reaction between creatinine and alkaline picrate. Glucose slowly reduces picric acid to picramate, while bilirubin, under alkaline 
conditions, is oxidized to biliverdin, causing a decrease in absorbance at $520 \mathrm{~nm}$. One of the most accurate routine methods is the enzymatic creatinine assay. It has been concluded by several studies that this method is suitable as a routine diagnostic laboratory method for the measurement of serum creatinine. Glucose does not interfere with the enzymatic method, while bilirubin causes a negative interference which depends on both creatinine and bilirubin concentrations. Comparison with the Jaffe's kinetic method showed that the enzymatic creatinine assay deals effectively with most interfering substances. However, it is more expensive and with a shorter shelf-life.

The aim of this study was to compare analytical performance and reliability of the enzymatic method and Jaffe's kinetic method in determination of serum creatinine for routine use, and to compare the effects of some common interfering substances like glucose and bilirubin on the enzymatic method and Jaffe's kinetic method.

\section{Materials and Methods}

The present study was conducted in the clinical laboratory in University Hospital - Pleven in 2014. We assessed 104 consecutive serum samples collected for routine clinical care.

Creatinine was analyzed by both Jaffe's kinetic and the enzymatic method. The Jaffe's method for serum creatinine determination is based on the principle that picric acid in an alkaline medium reacts with creatinine to form a yellow-red complex with the alkaline picrate [4]. The enzymatic method is based on the established determination of hydrogen peroxide after conversion of creatinine with the aid of creatinase and sarcosine oxidase.The liberated hydrogen peroxide reacts with 4aminophenazone and HTIB to form a quinone imine chromogen [5,6]. All measurements were performed using Cobas Integra 400 analyzer.

Quality control materials for creatinine on two levels (normal and pathological) were used. We also estimated serum total bilirubin by diazo method and serum glucose - by hexokinase method of the respective samples. The data obtained were divided into 3 groups. Group I comprised 37 samples without interfering substances (samples with serum glucose $<5.9$ $\mathrm{mmol} / \mathrm{l}$ and serum total bilirubin $\leqslant 17 \mu \mathrm{mol} / \mathrm{l}$ ). Group II comprised 36 samples with bilirubin (samples with serum total bilirubin $<17 \mathrm{Mmol} / 1$ and Serum Glucose $<5.9 \mathrm{mmol} / 1$ ); Group III comprised 31 samples with glucose (serum glucose $\geqslant 5.9 \mathrm{mmol} / \mathrm{l}$ and serum total bilirubin $\leqslant 17 \mathrm{Mmol} / \mathrm{l}$ ), and Group IV - all 104 samples .

We determined the difference between the two methods and analysed the agreement between them. The two methods were also compared by regression analysis. The two levels of quality control materials were analysed for precision by the enzymatic and Jaffe's kinetic methods. Statistical Package for the Social Sciences (SPSS, version 11.0) was used for statistical analyses. After analyzing data obtained using the two methods, the significance of differences between the methods was determined.

\section{Results}

In our study, estimation of creatinine by enzymatic method showed no statistically significant "p" value with the Jaffe's kinetic method (except in the normal group). In the presence of high glucose level, results from sample analyses showed no statistical significant difference between enzymatic and Jaffe's kinetic method. When bilirubin was present in the serum samples, the difference between enzymatic and kinetic Jaffe's method was also statistically insignificant. The results are presented in Table 1. The table shows the estimated parameters: Mean, SD and "p" value. In group I (normal), "p" value is $>0.10$. In the second group (high bilirubin), " $p$ " value is not significant $p=0.0305$. In groups III and IV, "p" values were not significant. (Table 1).

In Group IV (all samples), the correlation coefficient $(\mathrm{R}=0.983)$ was established after comparison between the enzymatic creatinine method (x) and kinetic Jaffe's method (y). All correlation coefficients for the two methods in the four groups are presented on Table 2 .

Correlation coefficients for both methods in all four groups are shown on Figures 1-4. 
Gencheva I., Ruseva A. Effects of glucose and bilirubin on the kinetic jaffe's and ...

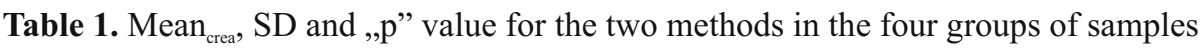

\begin{tabular}{|c|c|c|c|}
\hline Groups & Method & Mean $_{\text {crea }} \pm \mathbf{S D}(\mathrm{M} \mathrm{mol} / \mathrm{l})$ & 'p' value \\
\hline \multirow{2}{*}{$\begin{array}{l}\text { Group I } \\
\text { (Normal) }\end{array}$} & Enzymatic $(n=37)$ & $866.6 \pm 226.9$ & \multirow{2}{*}{$\mathrm{P}>0.10$} \\
\hline & Kinetic Jaffe's $(n=37)$ & $900.6 \pm 231$ & \\
\hline \multirow{2}{*}{$\begin{array}{l}\text { Group II } \\
\text { (Bilirubin) }\end{array}$} & Enzymatic $(n=36)$ & 72. $36 \pm 29.8$ & \multirow{2}{*}{$\mathrm{P}=0.0305$} \\
\hline & Kinetic Jaffe's (n=36) & $91.5 \pm 40.7$ & \\
\hline \multirow{2}{*}{$\begin{array}{l}\text { Group III } \\
\text { (Glucose) }\end{array}$} & Enzymatic $(\mathrm{n}=31)$ & $575.78 \pm 331.93$ & \multirow{2}{*}{$\mathrm{P}=0.0097$} \\
\hline & Kinetic Jaffe's $(n=31)$ & $603 \pm 333.77$ & \\
\hline \multirow{2}{*}{$\begin{array}{l}\text { All Groups } \\
\text { (Total) }\end{array}$} & Enzymatic $(n=104)$ & $534 \pm 396$ & \multirow{2}{*}{$\mathrm{P}<0.0001$} \\
\hline & Kinetic Jaffe's $(n=104)$ & $570 \pm 403$ & \\
\hline
\end{tabular}

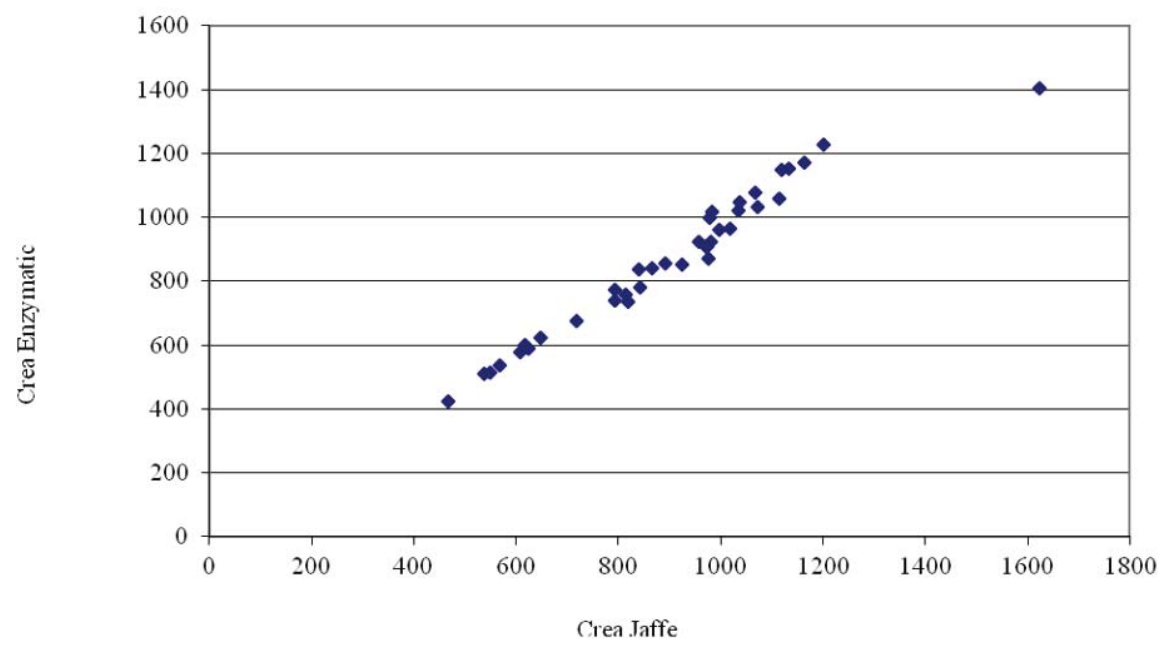

Figure 1. The correlation coefficients for the two methods in Group I

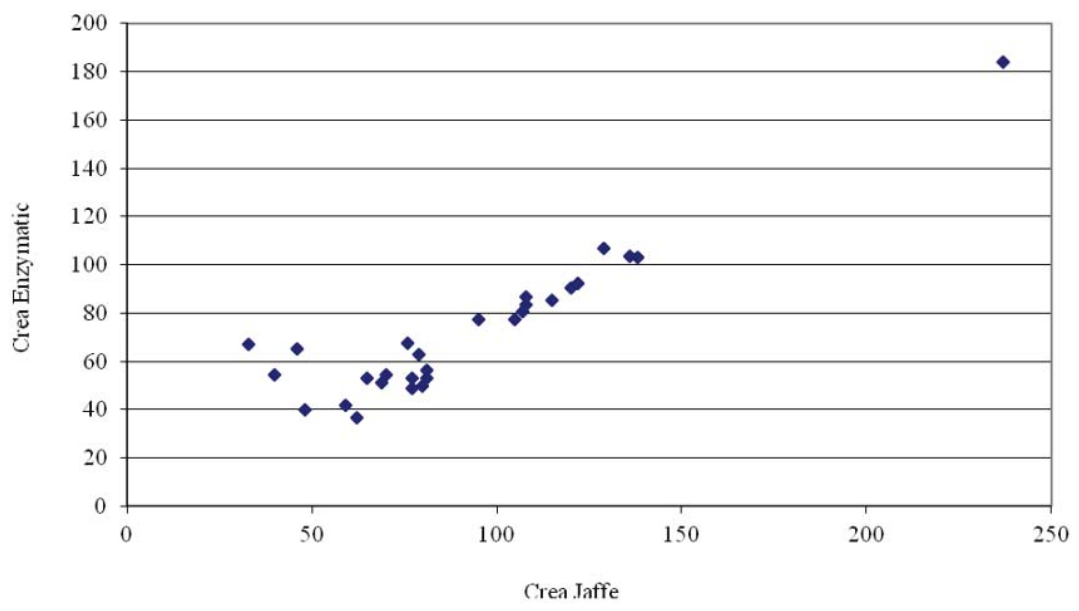

Figure 2. The correlation coefficients for the two methods in samples with high level of bilirubin 


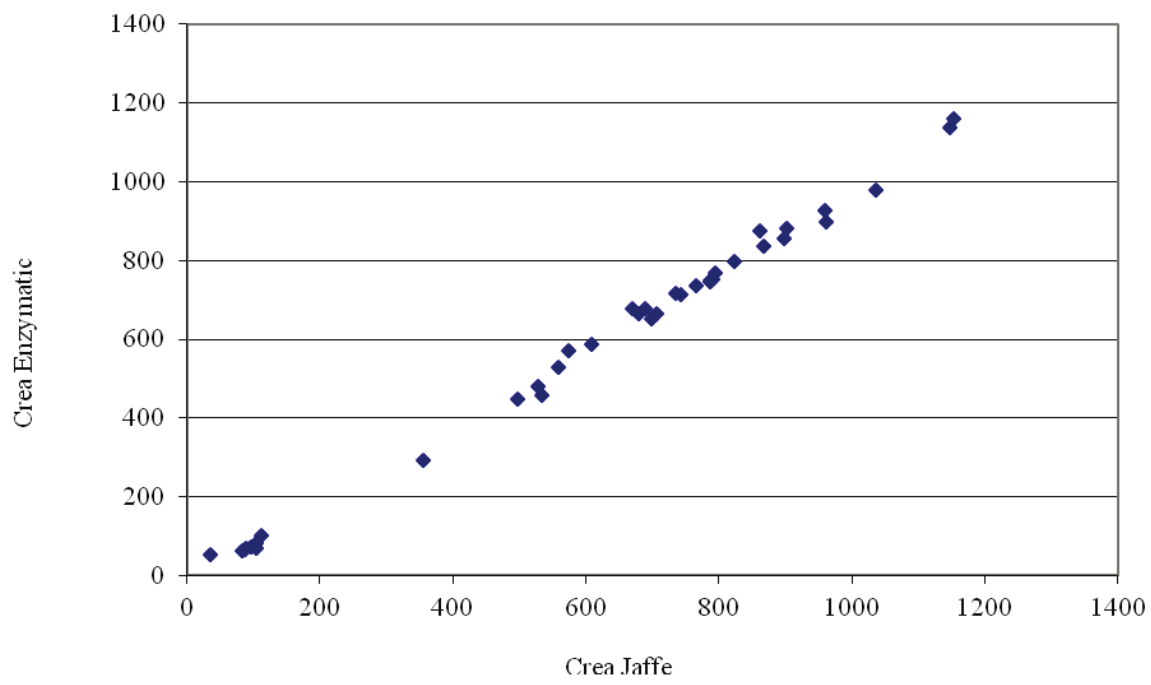

Figure 3. The correlation coefficients for the two methods in samples with high level of glucose

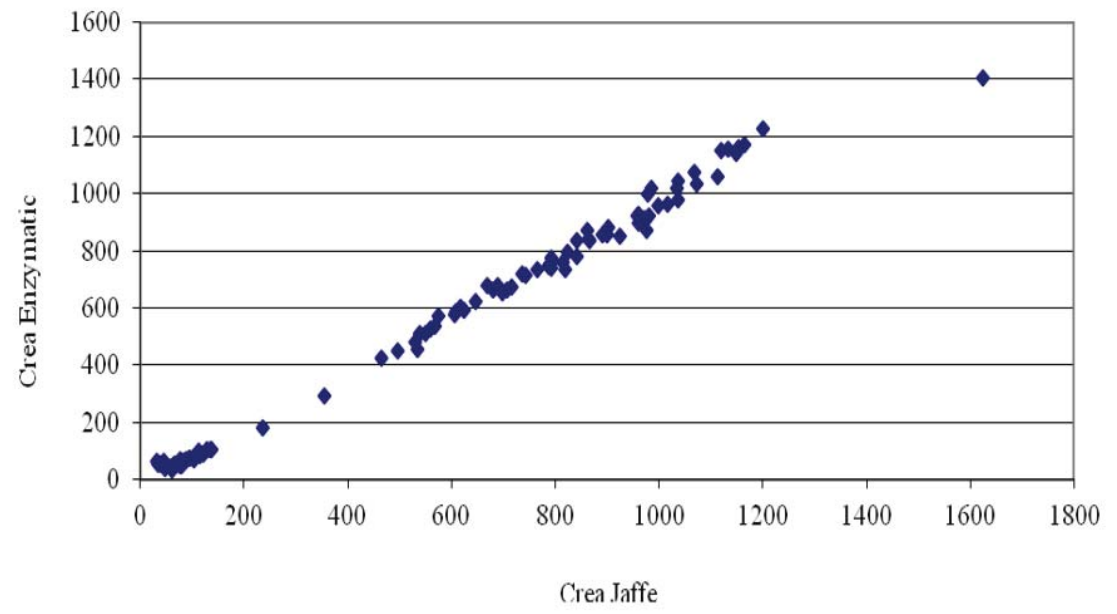

Figure 4. The correlation coefficients for two methods in all groups

Table 2. The correlation coefficients for the two methods in the groups

\begin{tabular}{ll}
\hline Groups & Correlation coefficient \\
\hline Group I (Normal) & 0.955 \\
\hline Group II (Bilirubin) & 0.971 \\
\hline Group III (Glucose) & 0.976 \\
\hline All Groups (Total) & 0.983 \\
\hline
\end{tabular}

\section{Discussion}

The enzymatic method was found to have certain advantages over Jaffe's based methods, and especially lack of interference with substances such as glucose and bilirubin. A minor disadvantage of the enzymatic method is its relatively higher cost. Use of Jaffe kinetic method yielded substantially higher values for creatinine, as compared with those obtained using the enzymatic method. These results are in accordance with several studies that compared the two methods. These results indicate that Jaffe methods, based on an alkaline picrate reaction, overestimate true serum creatinine concentrations, mainly due to non-specific interference. In our study, the correlation coefficients for the two methods in group I, Group II, Group III and Group IV (all samples), indicated a very good agreement between Kinetic Jaffe's method and enzymatic method (Table 2).

The results obtained proved a very good comparability between the two methods in all the 
settings specified: with or without the presence of interfering substances like glucose and bilirubin, as well as in the group comprising all the samples. Replacing the alkaline picrate method with the enzymatic creatinine method into routine laboratory practice is in accordance with recent recommendations of the Laboratory Working Group of the National Kidney Disease Education Program [7]. It is suggested that the estimated glomerular filtration rate has to be reported using accurate and specific serum creatinine measurements, based on the concept of traceability $[8,9]$.

\section{Conclusion}

There is good agreement between the kinetic Jaffe's method and the enzymatic method for estimation of creatinine levels. However, the enzymatic method is more reliable when interfering substances are present in the samples analysed, which makes is a method of choice.

\section{References}

1. Crocke H, Shephard S MD, White HG. Evaluation of an enzymatic method for determining creatinine in plasma. J Clin Pathol. 1988;41(5):576-81.

2. Peake M, Whiting M. Measurement of serum creatinine - Current status and future goals. Clin Biochem Rev. 2006;27(4):173-84.

3. Panteghini M, IFCC Scientific Division. Enzymatic assays for creatinine: time for action. Clin Chem LabMed. 2008;46(4):567-72.

4. Chromy V, Rozkosna K, Sedla KP. Determination of serum creatinine by Jaffe method and how to calibrate to eliminatematrix interference problems. Clin Chem Lab Med. 2008; 46(8):112733.

5. Cook JGH, Association of clinical Biochemists' Scientific and Technica Committee. Factors influencing the assay of creatinine. Ann Clin Biochem. 1975;12(6):219-32.

6. Spencer K. Analytical reviews in clinical biochemistry: the estimation of creatinine. Ann Clin Biochem. 1986;23(Pt 1):1-25.

7. Boyne P, Beilby J, Hickman P, Knight J, Williams D. Scientific Technical report No. 27 Creatinine Interferences Working Party. Clin Biochem Rev. 1988;9:54-7.

8. Twardowski ZJ, Nolph KD, Khanna R, Prowant BF, Ryan LP, Moore HL, et al. Peritoneal equilibration test. Perit Dial Bull. 1987;7:138-47.

9. Gerard S, Khayam-Bashi H. Negative interference with the Kodak Ektachem (Kodak) enzymic assay for creatinine by high serum glucose. Clin Chem. 1984;30(11):1884. 